0890-6955(95)00049-6

\title{
STUDY OF WIRE BREAKING PROCESS AND MONITORING OF WEDM
}

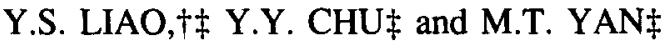 \\ (Received for publication 10 May 1995)
}

\begin{abstract}
Wire rupture in the Wire Electrical Discharge Machining (WEDM) process is a serious problem to manufacturers. A new computer-aided pulse discrimination system based on the characteristics of voltage waveform during machining was developed in this paper. With the use of this system, a large amount of sparking frequency data during wire rupture process and under normal working conditions were collected and analyzed. Two symptoms of wire rupture were identified: the excess of arc sparks, and a sudden rise of the total sparking frequency. The governing mechanisms of these two types of wire rupture were found from the SEM and EDAX analyses of the ruptured wire electrode. Furthermore, an index to monitor wire breaking was identified, and its relationships with the metal removal rate and machining parameters were found. Based on the results obtained in the paper, a control strategy to prevent wire from rupturing while at the same time improving the machining speed is proposed. (C) 1997 Published by Elsevier Science Ltd. All rights reserved
\end{abstract}

\section{INTRODUCTION}

The technologies of WEDM have grown rapidly in recent years owing to the requirement in various manufacturing fields. However, a few problems involved in WEDM are still far from solved. Among them, wire breaking during machining process is one of the serious problems in the application of WEDM. The occurrence of wire rupture would result in a great increase of machining time, a decrease of machining accuracy, and the deterioration of machined surface. Many researches on wire rupture have been carried out over the years with the objective of improving machining speed and machining accuracy. Kinoshita et al. [1] observed that the pulse frequency of gap voltage rose abruptly and lasted for approximately $5-40 \mathrm{~ms}$ before wire breakage. Based on this result, they developed a monitoring and control system. Once a sudden rise of pulse frequency was detected, the pulse generator and servo system was turned off instantaneously to prevent the wire from breaking. But when the power of the pulse generator is resumed, the pulse frequency of the gap voltage will generally increase suddenly, and shutting off the power is again necessary. The repeated on-off of the power affects the machining efficiency. Besides, the system was not suitable for workpieces of thickness less than $20 \mathrm{~mm}$. Similar to Kinoshita et al. [1], Rajurkar and Wang [2,3] found that wire breakage is closely related to the sparking frequency. They proposed a strategy to avoid wire rupture by controlling the sparking frequency at a constant level. However, the above approach is a passive means since the cutting speed is proportional to the sparking frequency, and lowering the sparking frequency would result in a decrease of metal removal rate. Jennes et al. [4] derived a thermal model to examine the relative influence of various process parameters on the thermal load of the wire electrode. They pointed out that temperature is the most prominent factor regarding wire breakage, and wire breakage would take place once the instantaneous energy rate exceeds a certain limit depending on the thermal properties of the wire material. Shoda et al. [5] found that the concentration of discharges at a certain point causes the wire breakage due to the increased local temperature. Based on this result, an adaptive control strategy for wire breakage prevention by decreasing the sparking energy was developed. All these studies contribute to the knowledge of wire rupture. But,

$\dagger$ Author to whom correspondence should be addressed.

$\ddagger$ Department of Mechanical Engineering, National Taiwan University, Taipei, Taiwan. 
WEDM is a very complex process, and the mechanism behind wire rupture is still not understood comprehensively.

Watanabe et al. [6] proposed a statistical pulse-classification method. The pulse trains are classified into three types: normal, deion and arc by statistical comparison of the collected gap voltage and current signals. The classified pulse is found to be able to reveal the gap condition. Hence, it can be applied to find the optimal current form so as to improve the machining stability and efficiency. In addition, it can be used to prevent wire from rupturing. However, the reliability of this kind of classification is questionable because it does not provide the physical meaning of each pulse. Nonetheless, it can be concluded that the discrimination of pulse types would provide more information about the WEDM process.

In general, the pulses in WEDM process can be classified into four signals, namely, open, normal, arc and short. Each kind of pulse has its own influence on wire electrode and workpiece. Concerning the effect on the mental removal rate, normal is the best pulse, arc is the next best one, while short and open are the worst pulses. As for their effects on wire electrode, many researchers have observed that an excess of short pulses resulted in wire breakage. But, the effects of other pulses remained unknown. Therefore, based on the reviews and discussion given above, the main purpose of this paper is to study the wire rupture mechanism. The relationship between the machining speed from the viewpoint of pulse discrimination will be investigated as well. A sparking frequency monitoring system based on pulse train analysis during machining process is introduced. A control strategy which can be applied to prevent wire from rupturing while at the same time improving the machining speed is proposed.

\section{PRINCIPLES OF PULSE GENERATION IN WEDM}

The iso-energy power supply circuit of WEDM developed by Industrial Technology Research Institute (ITRI), Taiwan is shown in Fig. 1. This circuit has the following characteristics:

(1) The gap current pulse is a triangular wave.

(2) Low power circuit leads the gap to discharge. This is followed by high power discharging $(220 \mathrm{~V})$ which contributes to metal removal.

The timing chart of pulse control signal is given in Fig. 2. The pulse control is accomplished by the following steps. The low power is turned on first by supplying $100 \mathrm{~V}$.

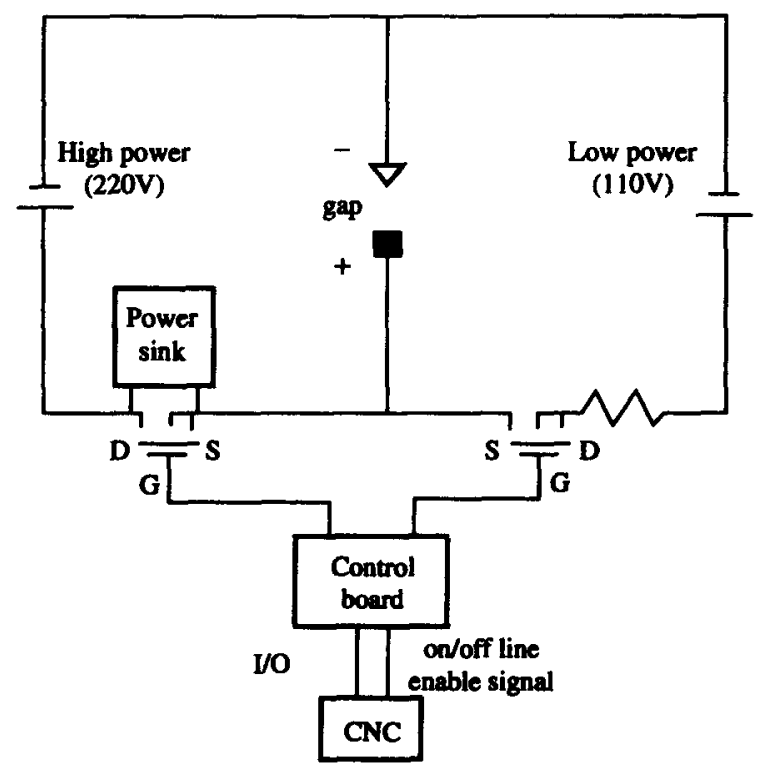

Fig. 1. WEDM power supply circuit. 


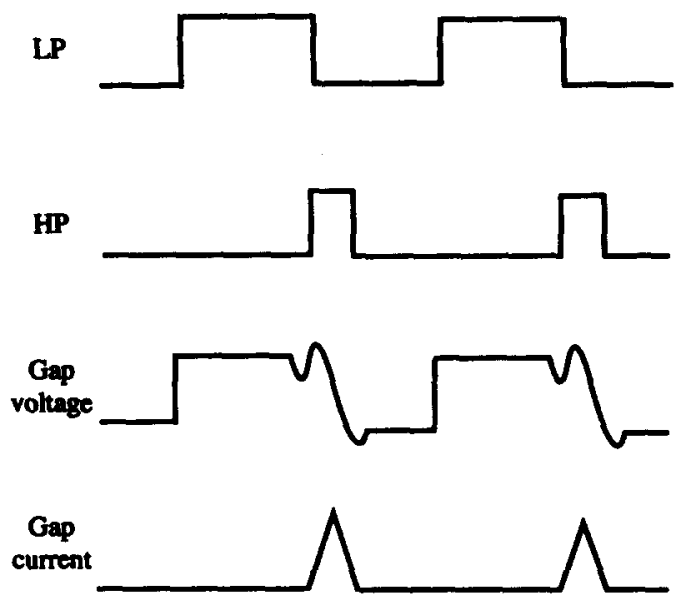

Fig. 2. Timing chart of the pulse control signal and the associated pulse trains of WEDM.

This causes the gap to discharge, but the gap voltage at this period is less than the preset value $(40 \mathrm{~V})$. At the second stage, the low power is turned off while the high power is turned on. At this moment, the gap is discharged with a high discharging current. After a short period of time $(<0.5 \mu \mathrm{s})$, the gap ceases to discharge. The gap remains in an insulated state until the low power is turned on again, and the next cycle starts.

The gap voltage and associated current waveforms are shown in Fig. 3. There are three sparks shown in the figure. The first one is a normal spark. The gap voltage begins at $80 \mathrm{~V}$, then the gap is discharged at a lower voltage with a high discharge current. After

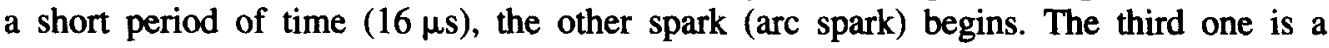
short spark.

\section{SPARKING FREQUENCY MONITORING CIRCUITS}

Typical timing charts and wave forms of normal and abnormal pulse control signals are shown in Figs 4(a) and 4(b), respectively. In the figures $L P$ and $H P$ are the control signals of the low and high power supplies, respectively. $V g$ is the gap voltage. $I g l$ and Igh are currents of the low and high power circuits, respectively. DMP is the detecting signal. INT is the internal signal which is used to judge if the spark is normal or abnormal. $t c$ and $t d l$ are rise time and rise time constant, respectively, and $t d 1>t c . t d 2$ is the time constant for the judgment of normal or abnormal sparks. $t$ th and $t s$ are delay times of the high power supply and the control signal, respectively, and $t s<150 \mathrm{~ns}$.

From Figs 4(a) and 4(b), the gap voltage $V g$ is the external signal used to judge if discharge had occurred, while INT is the internal signal used to check if the spark is normal or not. It is noted that under abnormal sparking conditions (arc or short), the gap

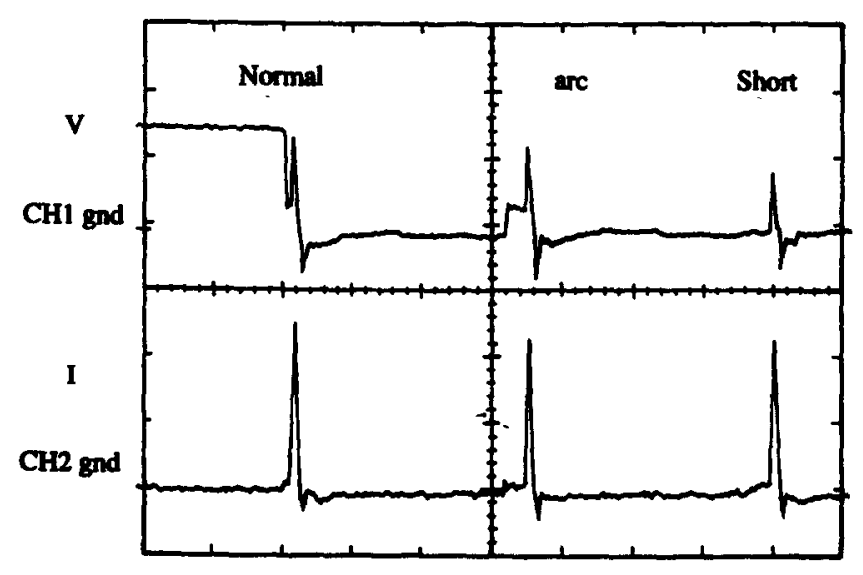

Fig. 3. Typical gap voltage and current waveforms of a WEDM process. 


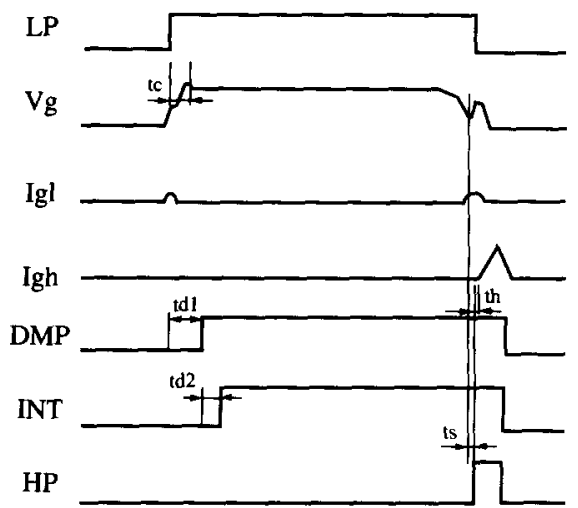

(a)

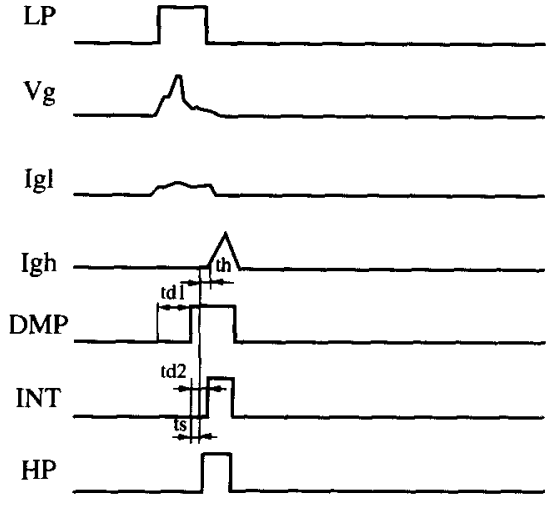

(b)

Fig. 4. Illustrations of timing chart to detect (a) the normal pulse signal, (b) the abnormal pulse signal.

voltage would drop abruptly, while under normal sparking conditions, $V g$ remains at a high level for a certain period of time. Hence, the system starts to monitor gap voltage when $D M P$ is changed from Low to High after a time span of $t d 1$. If the gap voltage is dropped within a time period pre-set by $I N T$, then it is taken as an abnormal spark by the control signal. But if the gap voltage drops after INT has changed from Low to High, then it is considered as a normal spark. Figure 5 shows the configurations of WEDM sparking frequency monitoring system. The voltage signal is sensed by the gap detecting circuit, made up of a distributing circuit and a comparator. The gap voltage together with $L P, I N T$ and $D M P$ mentioned previously, and the machining parameters such as on-time, off-time, arc on-time and arc off-time, etc. are input to the logic analysis circuit for the discrimination of normal and abnormal sparks. After logic analysis, the collected signals are classified into two types, namely, normal and arc discharge pulses, respectively. Due to the fact that there is a large interference of noises, and because it is difficult to differentiate the voltage level between arc and short sparks in practice, the short sparks are categorized in the arc pulse group. The normal pulse also consists of open sparks since the open sparks also discharge in the iso-energy power supply circuit, and the metal removal rate of a single discharge of open sparks is quantitatively equivalent to that of normal sparks. These pulses are converted to transistor-transistor logic (TTL) signals by photo couple circuit to filter high frequency noises. The total sparking frequency, and the sparking

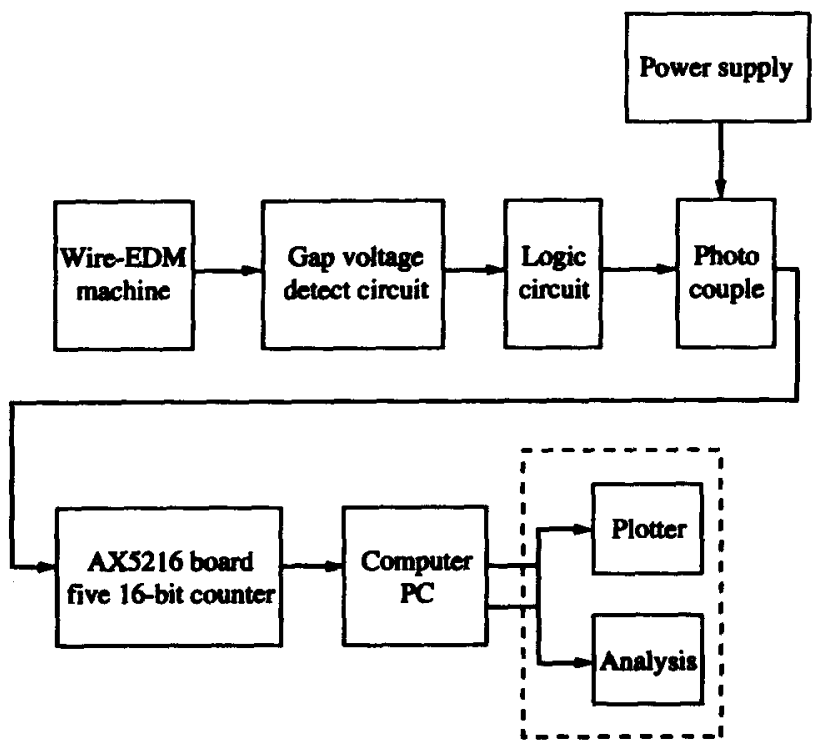

Fig. 5. WEDM sparking frequency monitoring system. 
frequencies of normal and arc pulses are collected continuously by an AX5216 counter card, and then they are stored into a file. The smallest sampling interval is limited by the record length, and the BAU rate between the PC's virtual disk and the AX5216 card. For our system, the record length is 20 bytes, and the sampling interval can not be smaller than $0.55 \mathrm{~ms}$. If the sampling interval is $10 \mathrm{~ms}$ and the virtual disk of PC486-25 is $4 \mathrm{Mb}$, then a long time data of $16 \mathrm{~min}$ can be collected continuously. Because of this long time data collection feature, the variations of sparking frequency before wire rupture can be exactly recorded to the PC's virtual disk. According to the result of some research $[1,2]$, the duration of the symptom of wire breakage is $20-50 \mathrm{~ms}$. Thus, the sampling interval of each data can not be set too long lest the change of sparking frequency before wire breakage should not be clearly detected. In this paper, 2-10 ms are chosen as the sampling time.

\section{PULSE TRAIN ANALYSIS UNDER NORMAL MACHINING CONDITIONS}

Figures 6(a), 6(b) and 6(c) show the sparking frequencies under normal machining conditions. The vertical and horizontal axes of these figures represent the sparking frequency $(\mathrm{kHz})$ and the machining time (s), respectively. In obtaining these data, the work material SKD11 of a thickness $50 \mathrm{~mm}$ is cut by a copper wire of $0.25 \mathrm{~mm}$ in diameter coated with a zinc $(\mathrm{Zn})$ layer. The machining parameters used are: on-time $=600 \mathrm{~ns}$, off-time $=9.6 \mu \mathrm{s}$, arc on-time $=300 \mathrm{~ns}$, arc off-time $=32 \mu \mathrm{s}$, wire speed $=5 \mathrm{~m} / \mathrm{min}$, wire tension $=1000 \mathrm{~g}$, machining speed $=19 \mathrm{~mm}^{2} / \mathrm{min}$, upper flushing pressure $=2 \mathrm{bar}$, lower flushing pressure $=1$ bar. The sampling interval used is $10 \mathrm{~ms}$. As the sampling time of this data is $10 \mathrm{~ms}$, the sparking frequency of $12 \mathrm{kHz}$ means there are 120 sparks in $10 \mathrm{~ms}$. In Fig. 6(a), the sparking frequency ranges from $10 \mathrm{kHz}$ to $16 \mathrm{kHz}$. By observing a few experiments, the sparking frequency distributes randomly with a small variation under a fixed machining condition and its mean value is kept almost at a fixed value. In these figures, the total sparking frequency, made up of the normal and arc sparking frequencies, ranges from $6 \mathrm{kHz}$ to $12 \mathrm{kHz}$ and its mean value is about $9 \mathrm{kHz}$. Figure 6 (d) shows the normal ratio defined as a ratio of the normal sparking frequency to the total sparking frequency. The normal ratio is kept at $70 \%$ during machining process. By contrast, the arc ratio defined as a ratio of the arc sparking frequency to the total sparking frequency is kept at $30 \%$ as shown in Fig. 6(e). Judging from a few experimental results, it is concluded that a good flushing condition (a good gap condition) would result in a normal ratio higher than $50 \%$.

\section{PULSE TRAIN ANALYSIS BEFORE WIRE RUPTURE}

\subsection{Wire rupture due to a sudden rise of total sparks (Type 1)}

After many experiments with various machining conditions, one type of symptom of wire breakage is found. Figures $7(\mathrm{a}), 7(\mathrm{~b})$ and $7(\mathrm{c})$ show the wire rupture process when the flushing pressure is decreased. In these figures, the total sparking frequency rises abruptly from $15 \mathrm{kHz}$ to $60 \mathrm{kHz}$, and the period of deterioration lasted for about $100 \mathrm{~ms}$. The variation in the arc sparking frequency is similar to that of the total sparking frequency. On the contrary, the normal sparking frequency ranges from $9 \mathrm{kHz}$ to $12 \mathrm{kHz}$ under a normal machining condition. But, it dropped rapidly to $6 \mathrm{kHz}$ after decreasing the flushing pressure, until the wire breaks. The deterioration process can be observed more clearly by examining the normal ratio and the arc ratio as those shown in Figs 7(d) and 7(e). The normal ratio is kept above $60 \%$ under a normal machining condition. However, it is decreased abruptly to far below $50 \%$ before wire rupture. Conversely, the arc ratio rises rapidly to above $50 \%$ during wire rupture process.

The machining parameters of the above data are given as follows: on-time: $500 \mathrm{ns,} \mathrm{off-}$ time: $25.6 \mu \mathrm{s}$, arc on-time: $300 \mathrm{~ns}$, arc off-time: $19.2 \mu \mathrm{s}$, wire speed: $5 \mathrm{~m} / \mathrm{min}$, wire tension: $1000 \mathrm{~g}$, work material: SKD11, thickness: $50 \mathrm{~mm}$, flushing pressure: upper $=1 \mathrm{bar}$, lower $=1$ bar, sampling interval: $10 \mathrm{~ms}$. 

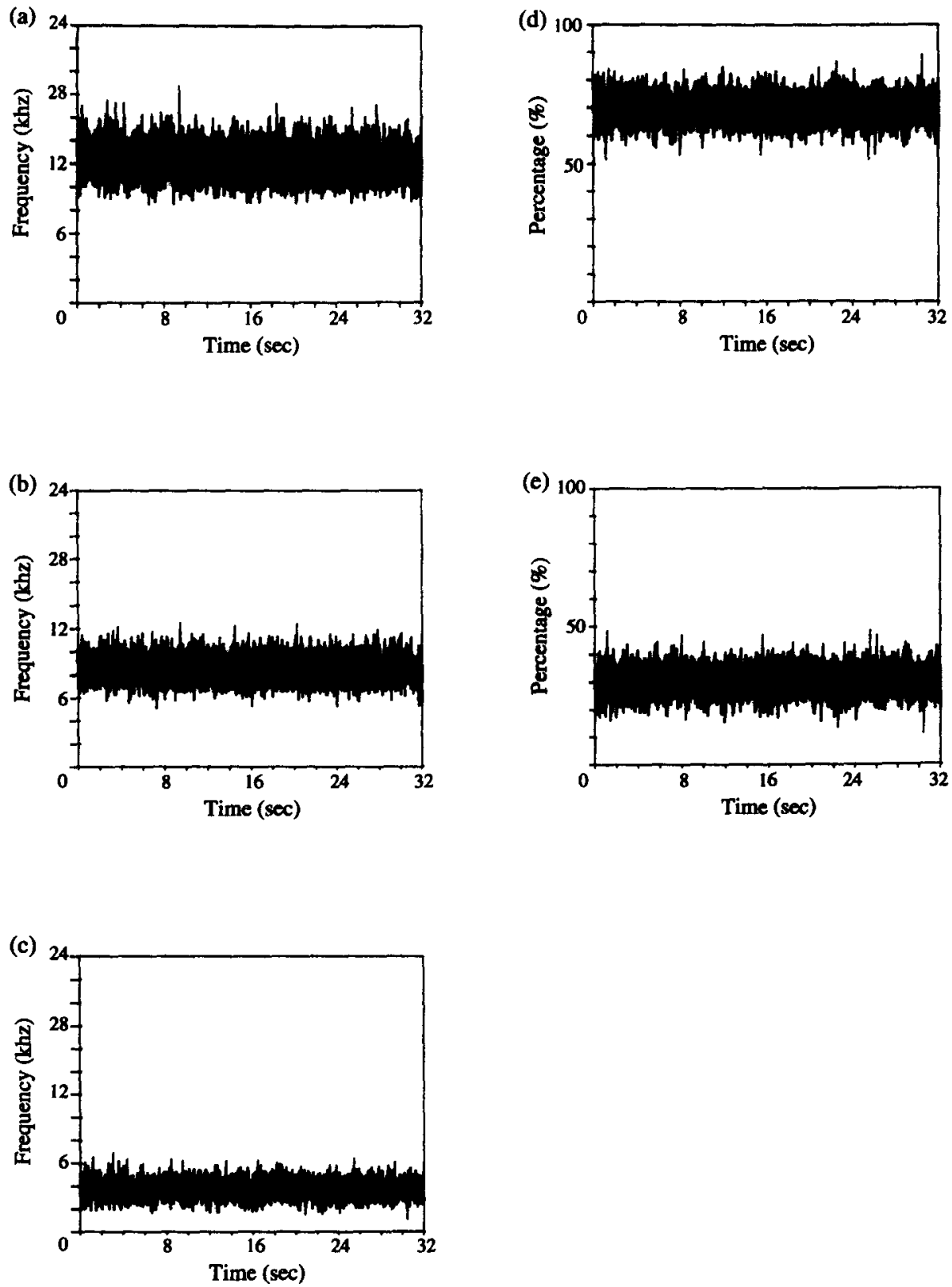

Fig. 6. (a) Total sparking frequency, (b) normal sparking frequency, (c) arc sparking frequency, (d) normal ratio and (e) arc ratio under normal machining conditions.

\subsection{Wire rupture due to an excess of arc sparks (Type 2)}

It often takes quite a long time for skilled operators of WEDM to complete the task when cutting a workpiece with complex shape. In order to prevent the wire electrode from breaking, they usually choose a set of relatively conservative machining parameters such as low discharging energy, low feedrate and high flushing pressure. However, this machining condition also results in wire breakage sometimes. It is a tiresome problem encountered by the operators. The results of a typical example when the cutting path has consecutive perpendicular corners are shown in Figs 8(a)-8(e). Looking at Fig. 8(d), the figure can be divided into two parts, the first part is under a normal machining condition, the normal ratio is kept at a level of about $75 \%$. The process starts to deteriorate in the second part where the normal ratio drops from $75 \%$ to below $50 \%$. The deterioration process proceeds until the wire is broken. By comparing the features reflected in the figure, the following facts are obtained: 

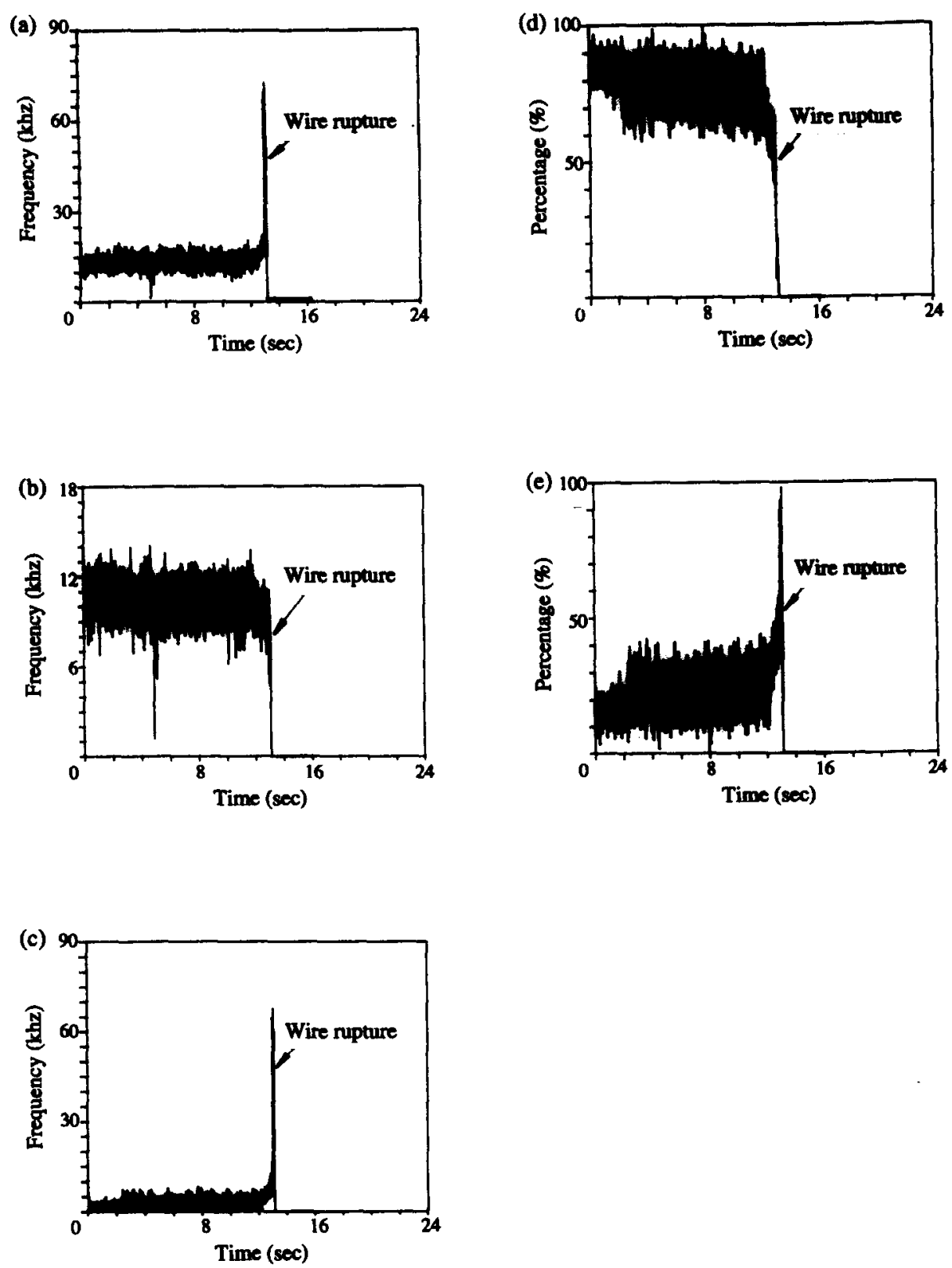

Fig. 7. (a) Total sparking frequency, (b) normal sparking frequency, (c) arc sparking frequency, (d) normal ratio and (e) arc ratio when the flushing pressure is decreased.

(1) The total sparking frequency remains at about $12 \mathrm{kHz}$ under normal machining conditions, this value is increased to $14-16 \mathrm{kHz}$ before wire rupture. Since there is not much difference in the total sparking frequency under normal machining conditions and before wire rupture, controlling of the total sparking frequency alone to avoid wire rupture is not applicable to this type of wire rupture.

(2) As compared with the normal sparking frequency, the arc sparking frequency rises more apparently during wire rupture process. As has been mentioned before, deterioration of the process can be assured when the normal ratio is dropped to below $50 \%$.

(3) The duration of the deterioration symptom lasted for about $60 \mathrm{~s}$ or more. But the rise of total sparking frequency is not apparent for this kind of wire breakage. Hence, gap condition can be identified not by the sudden rise of the total sparking frequency, but by the change of the normal ratio or the arc ratio.

Based on the above results, wire rupture may occur under normal machining conditions 

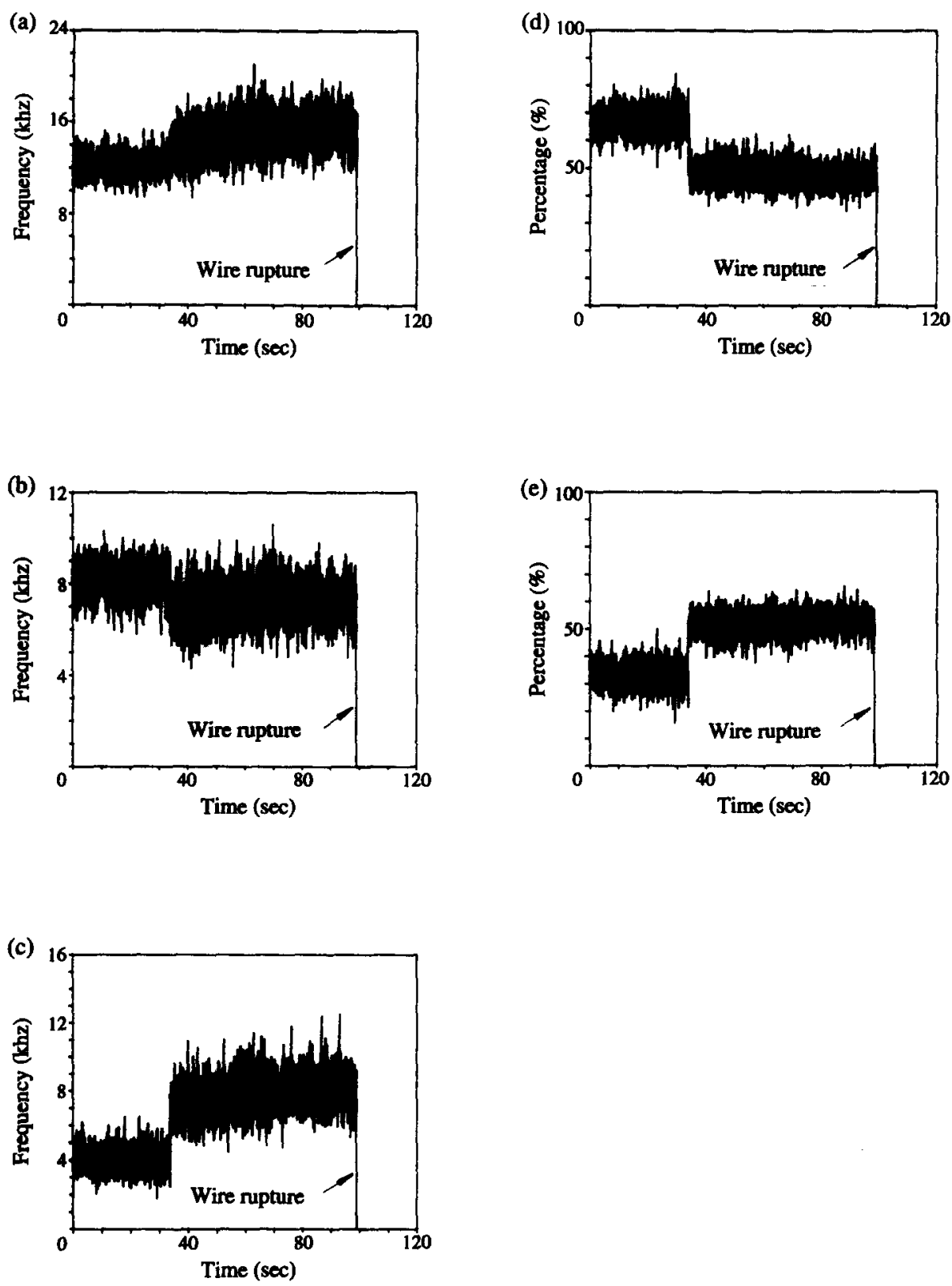

Fig. 8. (a) Total sparking frequency, (b) normal sparking frequency, (c) arc sparking frequency, (d) normal ratio and (e) arc ratio when a workpiece with complex shape is machined.

not because the wire electrode has absorbed too much discharging energy but because the gap condition has gradually deteriorated. The wire does not break instantaneously because of the continuous feed of wire electrode. But the gap remains deteriorated until the wire breaks.

It should be noted that both types of wire rupture would result in an increase of arc sparks. But the increase of arc ratio is larger for the second type of wire rupture. The study of Kunieda and Kojima [7] had concluded that the uneven distribution of spark locations prefigures an arc. Hence, when the wire rupture occurs, the discharges are expected to concentrate at a certain point as observed by Shoda et al. [5].

\section{MACRO- AND MICRO-STRUCTURES STUDY OF WIRE ELECTRODE}

As mentioned in the previous section, there are two symptoms of wire rupture. One is the sudden rise of the total sparking frequency, the other is the excess of the arc sparks. 
In this section, the mechanisms behind these two types of wire rupture are investigated by SEM and EDAX analyses of the ruptured wire electrode. Figures 9(a) and 9(b) show the SEM photomicrographs of the end and side views of the ruptured wire electrode caused by the excess of the arc sparking frequency (Type 2 wire breakage). As shown in Fig. 9(a), there are many different craters on the end section of the ruptured wire. Some residuals adhered to the surface of wire electrode (bright spots in Fig. 9(b)). Analyzed by EDAX, the residuals are found to consist of $\mathrm{Cu}, \mathrm{Zn}, \mathrm{Fe}$, etc. The relative ratio of each composition is given in Fig. 10. Among the compositions, $\mathrm{Cu}$ and $\mathrm{Zn}$ are the elements belonging to the wire electrode. While $\mathrm{Fe}$ is one of the elements of workpiece. A badly insulated condition between the wire and the workpiece or a narrower gap is responsible for the residuals to adhere to the wire surface. Based on the above analysis, it is believed that under a badly insulated or a narrower gap condition, the arc sparks increase, the residuals of workpiece can not be flushed away efficiently by the dielectric. Thus, they adhere to the wire surface. In addition, since the gap is too narrow, a portion of discharging energy is absorbed by the wire electrode. Hence, the surface of wire melts due to the induced high temperature. Because the wire is subjected to a fixed tension during the cutting process, the melting of wire surface results in a smaller diameter of the wire electrode. This, in turn, causes the tensile stress of the wire electrode to rise rapidly until at last the wire breaks. Therefore, the ductile fracture results in many different craters to form on the end surface of the ruptured wire.

A SEM photomicrograph of the ruptured wire electrode which could be due to an excess of short sparks is given in Fig. 11. A very serious problem of the materials adhering on the end surface of the broken wire can be seen in the figure. Some contacts beween the wire and the workpiece or the bad condition results in too many arc sparks. Under this
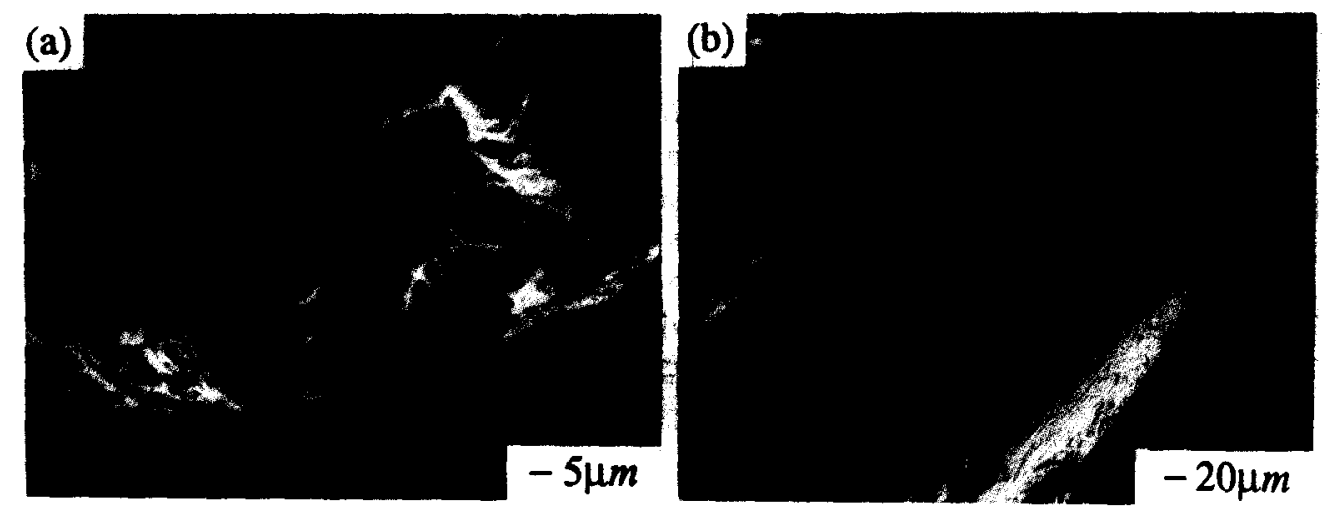

Fig. 9. SEM photomicrographs of the Type 2 ruptured wire: (a) end view, (b) side view.

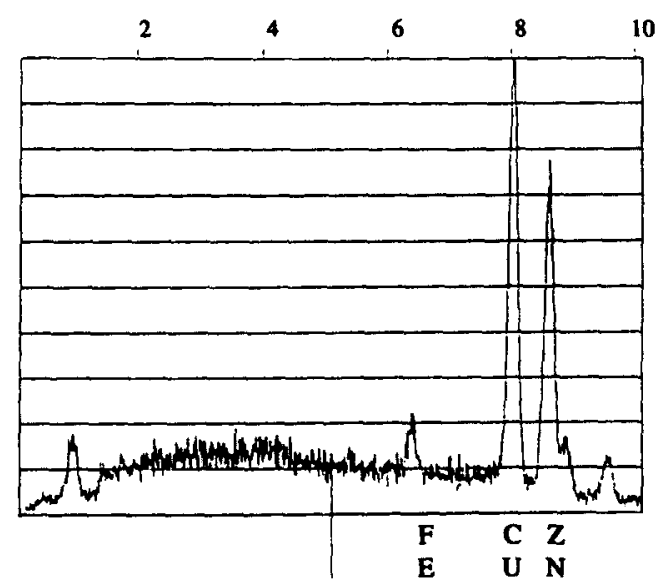

Fig. 10. EDAX analysis of the Type 2 ruptured wire. 


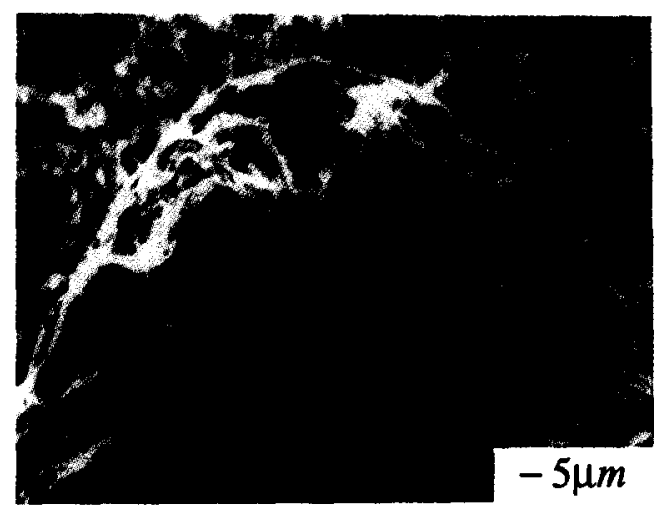

Fig. 11. SEM photograph of the side view of the ruptured wire due to the excess of short sparks.

condition, the residuals are left on the wire surface which causes a serious adhesion problem to take place.

Figures 12(a) and 12(b) show the SEM photomicrographs of the end and side views of the ruptured wire electrode due to a sudden rise of total sparking frequency (Type 1 wire breakage). From these figures, it can apparently be seen that this type of wire breakage is different from that mentioned before. There are two main differences. Firstly, there are no craters due to the ductile fracture on the end surface of the ruptured wire. Secondly, there are very few adhering materials on the wire. The reasons and process of this type of wire rupture are inferred as follows. When there is an excessive increase of the total sparks, the discharging energy rises quickly. Under such a condition, the working fluid can not effectively cool the wire. As a result, the wire electrode absorbs too much energy which leads to the rise of wire temperature. As the temperature is higher than a critical value the wire breaks. It is also noted that since the temperature is too high, the residual materials that adhere on the wire electrode are evaporated, and hence the adherence of residual materials on wire electrode is seldom observed.

The foregoing discussions can also be verified by observing the end surface of the ruptured wire. The surface of the wire electrode looks black when the breakage of the wire is caused by an excess of arc sparks, while the surface looks shiny for the rupture of wire due to a sudden rise of total sparking frequency.

\section{RELATIONSHIPS OF NORMAL RATIO WITH METAL REMOVAL RATE AND MACHINING PARAMETERS}

The previous study has revealed that the normal ratio can be taken as a parameter to judge the machining state as far as wire breakage is concerned. From the control viewpoint, wire breaking should be avoided, the machining speed should be improved (i.e. maximize
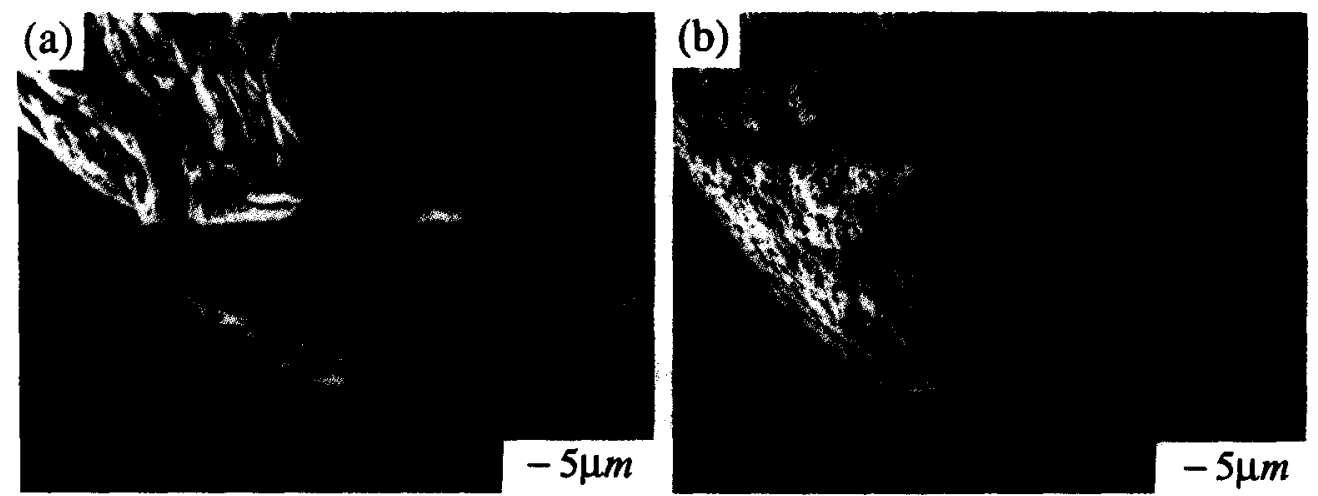

Fig. 12. SEM photomicrographs of the Type 1 ruptured wire: (a) end view, (b) side view. 
the metal removal rate) as well. Figure 13 shows the relationship of normal ratio with the metal removal rate, feed rate and gap voltage. From this figure, it can be seen that there exists an inverse relationship between the normal ratio and feed rate. The metal removal rate is maximum when the normal ratio is $46 \%$. But under this machining condition, the machining process is in a dangerous state of wire breaking. For our machine, a very simple wire breaking protection function based on the counting of successive abnormal sparks has been implemented by the machine tool builder. The table is withdrawn and then the feed is commenced again instantaneously by the motion system of the WEDM machine when the total number of successive abnormal sparks reaches a specific value. This is the reason why the metal removal rate shown in the figure is reduced when the machining process falls in the wire rupture region. Without this protection function the wire will break once the normal ratio is less than $40 \%$. However, it should be noted that even with this protection function, wire breakage still occurs from time to time. The experimental results given in the previous sections are typical examples that the wire breaking protection function fails. Based on Fig. 13, it is clear that the normal ratio should be kept in the range of $50-60 \%$ in order that a stable machining and high metal removal rate condition can be maintained while preventing the wire from breaking.

\section{STRATEGY TO AVOID WIRE RUPTURE}

According to the symptoms reflected in the variations of pulse trains during the wire rupture process, and the structures of the ruptured section of the wire, two types of wire rupture are categorized.

The adjustable parameters of this machine are listed in Table 1. These parameters are classified into four orders according to their response. Based on the results obtained in this paper and the experiences in WEDM, a control scheme is proposed to prevent wire

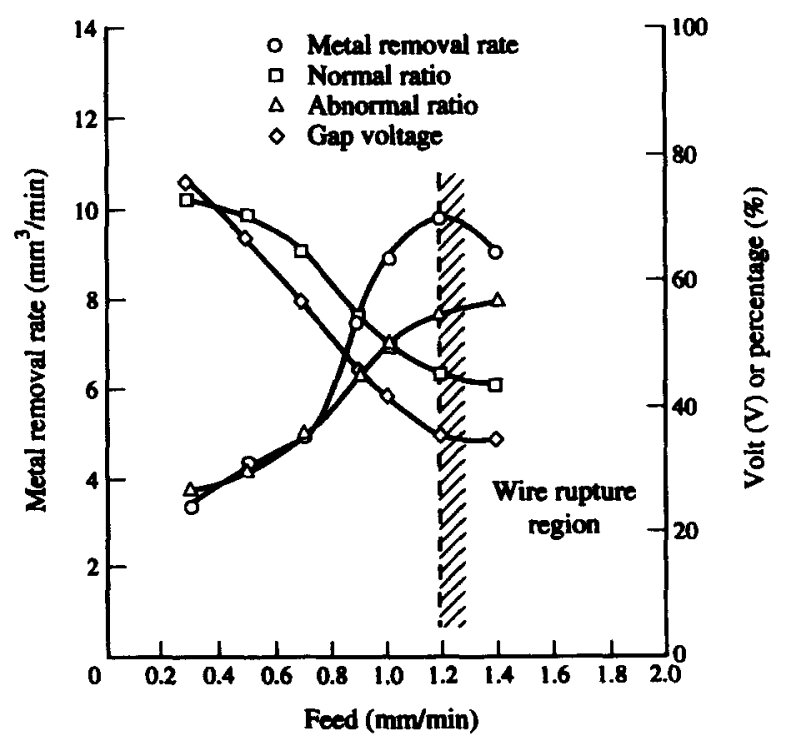

Fig. 13. Relationships of normal ratio with metal removal rate and machining parameters.

Table 1. Adjustable parameters of various orders in WEDM process

\begin{tabular}{lc}
\hline Order & Items \\
\hline Microsecond & $\begin{array}{c}\text { on time, arc on time, off time } \\
\text { arc off time, on time base } \\
\text { servo voltage, feedrate } \\
\text { Millisecond }\end{array}$ \\
$\begin{array}{l}\text { wecond } \\
\text { Minute }\end{array}$ & water conductivity, water pressure \\
\hline
\end{tabular}


rupture while improving the machining speed as well. Figure 14 illustrates the flow chart of the scheme. To apply this strategy, the collected sparking frequency is passed through a low pass Butterworth filter first to remove high frequency signals. If the resultant total sparking frequency $(y)$ is more than the reference frequency $(Y)$, the discharge parameters at the "microseconds" order should be adjusted immediately owing to a sudden rise of discharging energy and a very short period of deterioration. In addition, the feed rate should be adjusted to a lower value to avoid wire rupture as well. If the normal ratio $(\psi)$ is less than the reference ratio $(\Psi)$, a bad gap condition is encountered. Since the deterioration process lasted for a relatively long period of time, the parameters such as servo voltage, water pressure, water conductivity and feed rate which have long time response on the process should be adjusted at once to cope with the bad gap condition. In both cases, the normal ratio should be kept within a certain range (in our case it is 50-60\%) so that high metal removal rate can be achieved.

\section{CONCLUSIONS}

This paper develops a new computer-aided pulse discrimination system which can be employed to collect the sparking frequency of pulse trains for a long period of time. Thus, the variations of pulse frequency can be precisely recorded. Two types of wire rupture

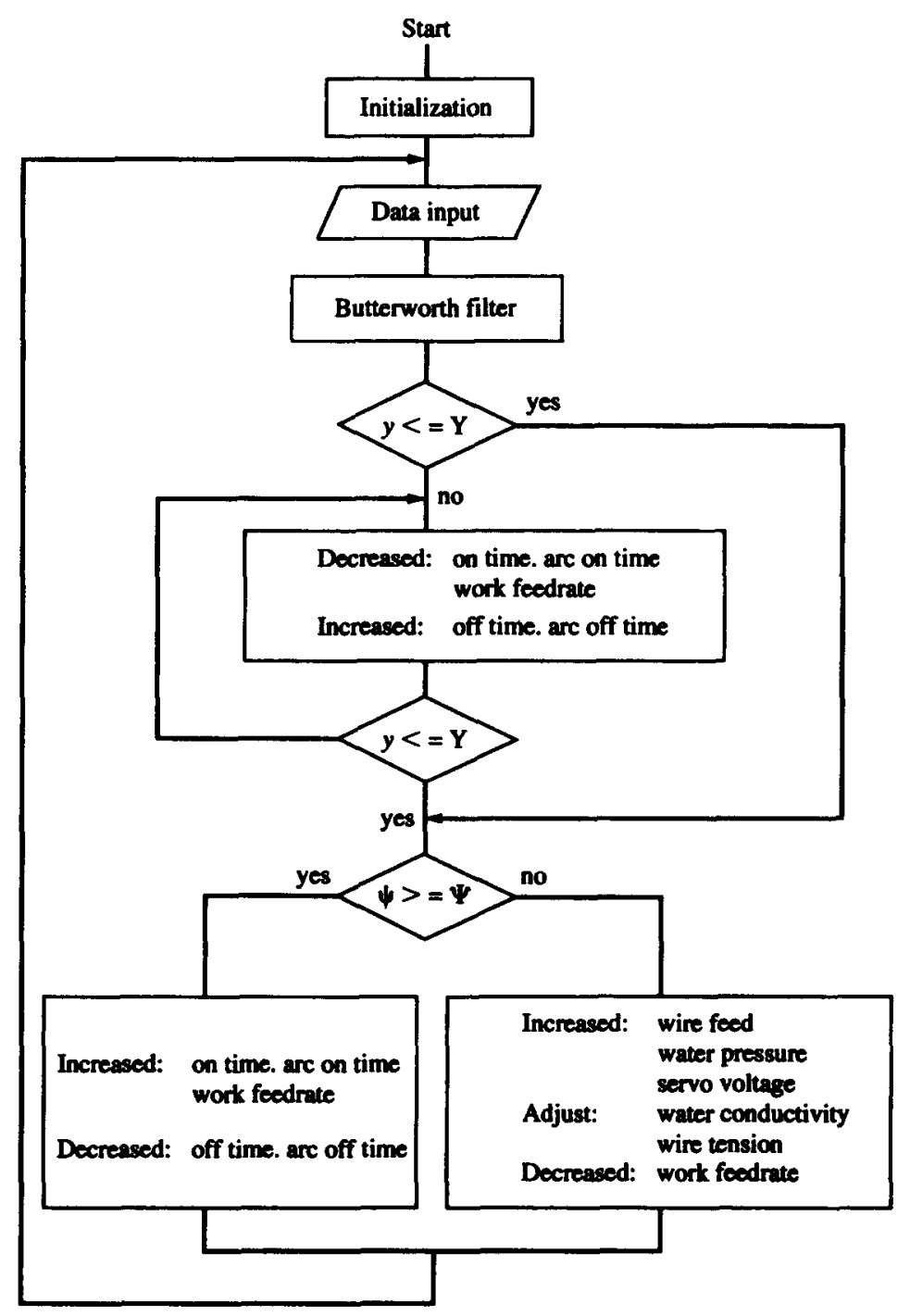

Fig. 14. Flow chart of the control program. 
are classified according to the observation of cross section of ruptured wire by SEM, and the symptoms of pulse trains during wire rupture process. An index to monitor the wire rupture is found. In addition, the relationships between this index, metal removal rate and machining parameters are found, and a control strategy to prevent wire rupture while improving the machining speed is proposed. The implementation of the proposed control strategy is under way.

\section{REFERENCES}

[1] N. Kinoshita, M. Fukui and G. Gammo Ann. CIRP 31, 111-114 (1982).

[2] K. P. Rajurkar and W. M. Wang Ann. CIRP 40, 219-222 (1991).

[3] K. P. Rajurkar and W. M. Wang Proc. 1992 Pacific Conf. on Manuf. 346-353 (1992).

[4] M. Jennes, W. Dekeyser and R. Snoeys Ann. CIRP 33, 93-98 (1984).

[5] K. Shoda, Y. Kaneko, H. Nishimura, M. Kunieda and M. X. Fan ISEM 10, 410-416 (1992).

[6] H. Watanabe, T. Sato and I. Suzuki Ann. CIRP 39, 175-178 (1990).

[7] M. Kunieda and H. Kojima Ann. CIRP 39, 171-174 (1990). 